\title{
Glutathione inhibits expression of the proinflammatory biomarker inducible nitric oxide synthase in hepatocytes
}

Richi Nakatake $^{1 *}$, Masaya Kotsuka ${ }^{1}$, Yuki Hashimoto ${ }^{1}$, Masahiko Hatta ${ }^{1}$, Morihiko Ishizaki ${ }^{1}$, Kosuke Matsui $^{1}$, Masaki Kaibori ${ }^{1}$, Mikio Nishizawa ${ }^{2}$ and Tadayoshi Okumura ${ }^{1,3}$

${ }^{1}$ Department of Surgery, Kansai Medical University, Hirakata, Osaka, Japan; ${ }^{2}$ Department of Biomedical Sciences, College of Life Sciences, Ritsumeikan University, Kusatsu, Shiga, Japan; ${ }^{3}$ Research Organization of Science and Technology, Ritsumeikan University, Kusatsu, Shiga, Japan

Corresponding author: Richi Nakatake, M.D., Ph.D. Department of Surgery, Kansai Medical University, 2-5-1 Shinmachi, Hirakata, Osaka, 573-1010, Japan.

Submission Date: August 22 ${ }^{\text {nd }}, 2018$, Acceptance Date: October $28^{\text {th }}, 2018$, Publication Date: December $30^{\text {th }}, 2018$

Citation: Nakatake R., Kotsuka M., Hashimoto Y., Hatta M., Ishizaki M., Matsui K., Kaibori M., Nishizawa M., Okumura T. Glutathione inhibits expression of the proinflammatory biomarker inducible nitric oxide synthase in hepatocytes. Functional Foods in Health and Disease 2018; 8(12): 544-560. DOI: https://doi.org/10.31989/ffhd.v8i11.547

\begin{abstract}
Background: Intracellular glutathione (GSH) plays an important regulatory role in the host response to liver injury. However, there have been few scientific reports on the anti-inflammatory effects of GSH. In the inflamed liver, proinflammatory cytokines stimulate liver cells, followed by expression of inducible nitric oxide synthase (iNOS). Excessive nitric oxide (NO) levels produced by iNOS are one of the factors involved in liver injury. Therefore, inhibiting iNOS induction is important for preventing liver injury. This study aimed to investigate the protective effects of GSH on the liver by examining interleukin (IL)-1 $\beta$-stimulated hepatocytes.
\end{abstract}

Methods: Primary cultured rat hepatocytes were treated with IL-1 $\beta$ in the presence or absence of GSH. Induction of iNOS and its signaling pathway were analyzed.

Results: Addition of GSH decreased IL-1 $\beta$-induced iNOS protein and mRNA expression levels, which resulted in inhibition of NO production. GSH also decreased tumor necrosis factor (TNF)- $\alpha$ and IL-6 mRNA expression. GSH blocked "type I IL-1 receptor upregulation", 
one of the essential signaling pathways for iNOS induction, through inactivation of an upstream kinase, phosphatidylinositol 3-kinase/Akt. In contrast, GSH had no effects on degradation of $\mathrm{I} \kappa \mathrm{B}$ and activation of NF- $\mathrm{KB}$ (nuclear translocation and its DNA binding). Transfection experiments revealed that GSH reduced iNOS mRNA levels at the promoter transactivation and mRNA stabilization steps. Delayed administration of GSH after IL-1 $\beta$ addition also inhibited iNOS induction.

Conclusions: Our study suggests that GSH affects induction of inflammatory mediators, including iNOS and TNF- $\alpha$, indicating its therapeutic potential for organ injuries, especially for the liver.

Keywords: glutathione, inducible nitric oxide synthase, liver injury, primary cultured hepatocytes, type I interleukin-1 receptor, tumor necrosis factor- $\alpha$

\section{BACKGROUND}

Glutathione (GSH), which is a sulfhydryl group-containing tripeptide (L-glutamyl-L-cysteinyl-glycine), is the most prominent intracellular low-molecular weight thiol (intracellular levels are in the $\mathrm{mM}$ concentration range) [1]. GSH serves as an important intracellular water-soluble antioxidant and detoxifying agent $[2,3]$. In addition to its strong antioxidant effect, GSH plays a crucial role, particularly in maintenance and regulation of the thiol redox status of the cell. These effects appear to correlate with proliferation or differentiation of lymphocytes, macrophages, and dendritic cells [4-9]. GSH-based hepatoprotective therapy can partially alleviate liver injury [10], although the mechanisms involved in the action of GSH are unclear.

In hepatic disorders, inflammatory cells such as macrophages gather around hepatic stellate (Kupffer) cells and discharge a variety of cytokines. During inflammation, proinflammatory cytokines and nitric oxide (NO) play an important role as factors in liver injury [11]. NO is produced by inducible nitric oxide synthase (iNOS) in Kupffer cells and hepatocytes. Expression of iNOS is enhanced in the liver after feeding a high fat diet $[12,13]$. Because of the critical role of iNOS in development of liver fibrosis, long-term inhibition of iNOS may have therapeutic benefits [14].

In animal liver injury models caused by various insults, such as ischemia-reperfusion, partial hepatectomy, and endotoxin shock, we found that drugs [15-19] and functional foods [20] demonstrated liver-protective effects. These drugs and functional foods led to higher survival rates and a reduction in various cytokine production, such as tumor necrosis factor (TNF)- $\alpha$, interleukin (IL)-1 $\beta$, IL-6, and cytokine-induced neutrophil chemoattractant (CINC)-1 (human IL-8 analogue) [15-19]. These clinical drugs [17, 21, 22, 23] and others, such as L-glutamic acid (component of GSH) [24], curcumin [25], and active hexose correlated compound [26], inhibited induction of iNOS and NO production in primary cultures of rat hepatocytes stimulated by IL-1 $\beta$ (“in vitro liver injury model”). Therefore, 
inhibition of iNOS induction and NO production in the "in vitro liver injury model" is an indicator of liver protection [27].

In the present study, we examined whether GSH affects induction of iNOS in IL-1 $\beta$-stimulated hepatocytes and the mechanisms involved in the action of GSH.

\section{METHODS}

Materials: GSH (Choseido Pharmaceutical Co., Ltd, Tokushima, Japan) was dissolved in Williams' medium E (WE) and used in experiments. Recombinant human IL-1 $\beta\left(2 \times 10^{7}\right.$ U/mg protein) was purchased from My Bio Source (San Diego, CA, USA). Male Wistar rats (200-250 g, 6-7 weeks old) were purchased from Charles River (Tokyo, Japan), kept at $22^{\circ} \mathrm{C}$ under a 12:12 h light: dark cycle, and received food and water ad libitum. All experiments with rats were performed in accordance with the Guidelines for the Care and Use of Laboratory Animals of the National Institutes of Health. The experiments were approved by the Animal Care Committee of Kansai Medical University.

Primary hepatocyte culture: Hepatocytes were isolated from rats by perfusion with collagenase (Wako Pure Chemicals, Osaka, Japan) [28]. Isolated hepatocytes were suspended in culture medium at $6 \times 10^{5}$ cells $/ \mathrm{mL}$, seeded into $35-\mathrm{mm}$ plastic dishes $(2 \mathrm{~mL} / \mathrm{dish}$; Falcon Plastic, Oxnard, CA, USA) and cultured at $37^{\circ} \mathrm{C}$ in a $\mathrm{CO}_{2}$ incubator under a humidified atmosphere of $5 \% \mathrm{CO}_{2}$ in air. The culture medium (WE) was Williams' medium $\mathrm{E}$ supplemented with $10 \%$ newborn calf serum, HEPES $(5 \mathrm{mmol} / \mathrm{L})$, penicillin $(100 \mathrm{U} / \mathrm{mL})$, streptomycin $(100 \mu \mathrm{g} / \mathrm{mL})$, amphotericin B $(0.25 \mu \mathrm{g} / \mathrm{mL})$, aprotinin $(0.1 \mu \mathrm{g} / \mathrm{mL}$; Roche, Mannheim, Germany), dexamethasone (10 nmol/L), and insulin (10 nmol/L). After $2 \mathrm{~h}$, the medium was replaced with fresh serum-free Williams' medium E (1.5 mL/dish). After $5 \mathrm{~h}$, the medium was replaced with fresh serum and hormone-free Williams' medium E (1.5 mL/ dish). Cells were cultured overnight before use. The number of cells attached to the dishes was calculated by counting the number of nuclei [29] and using a ratio of $1.37 \pm 0.04$ nuclei/cell (mean $\pm \mathrm{SE}, \mathrm{n}=7$ experiments).

Treatment of cells with GSH: On day 1, the cells were washed with fresh serum and hormone-free WE and incubated with IL-1 $\beta(1 \mathrm{nM})$ in the same medium in the presence or absence of GSH. The doses of GSH used are indicated in the appropriate figures and their legends.

Determination of NO production: The culture medium was used to measure nitrite (a stable metabolite of NO) levels, which reflect NO production, using the Griess method [30].

Western blot analysis: As described previously, total cell lysates were obtained from cultured cells with minor modifications [26]. Briefly, cells $\left(1 \times 10^{6}\right.$ cells/35-mm dish) were lysed with sample buffer for sodium dodecyl sulfate-polyacrylamide gel electrophoresis 
(SDS-PAGE) (final: $125 \mathrm{mM}$ Tris-HCl, $\mathrm{pH}$ of 6.8; containing 5\% glycerol, 2\% SDS, and 1\% 2-mercaptoethanol), subjected to SDS-PAGE, and electroblotted onto a polyvinylidene difluoride membrane (Bio-Rad, Hercules, CA, USA). Immunostaining was performed using primary antibodies against mouse iNOS (Affinity BioReagents, Golden, CO, USA), human IкB $\alpha$, mouse type I IL-1 receptor (IL-1RI) (Santa Cruz Biotechnology, Santa Cruz, CA, USA), and rat $\beta$-tubulin (internal control, Clone TUB2.1; Sigma Chemical Co., St. Louis, MO, USA). This was followed by visualization with an enhanced chemiluminescence blotting detection reagent (GE Healthcare Biosciences Corp., Piscataway, NJ, USA).

For Akt, total cell lysates that were prepared from $100-\mathrm{mm}$ dishes $\left(5 \times 10^{6}\right.$ cells/dish $)$ were pre-cleared with protein A (Sigma Chemical Co.) and then mixed with a mouse monoclonal antibody against human Akt1 (Akt5G3; Cell Signaling, Beverly, MA, USA) and protein G-Sepharose (Pharmacia LKB Biotech, Uppsala, Sweden). After incubation overnight at $4^{\circ} \mathrm{C}$, immunocomplexes were centrifuged $(16,000 \times g$ for $5 \mathrm{~min})$. The beads were washed with solubilizing buffer and dissolved in SDS-PAGE sample buffer. Analysis by western blotting was then performed using rabbit polyclonal antibodies against human Akt and phospho-Akt (Ser473) (Cell Signaling) as primary antibodies. For p65, nuclear extracts were immunoprecipitated with an anti-p65 antibody (H286; Santa Cruz Biotechnology). The bands were analyzed by western blotting using an antibody against human nuclear factor-kappa B (NF-кB) p65 (BD Transduction Laboratories, Lexington, KY, USA).

Real-time reverse transcriptase-polymerase chain reaction: Total RNA was extracted from cultured hepatocytes using the guanidinium-phenol-chloroform method [31]. For strand-specific reverse transcriptase-polymerase chain reaction (RT-PCR) analysis, cDNA was synthesized from total RNA using strand-specific primers. Step-down PCR was performed using PC708 (Astec, Fukuoka, Japan) as previously described [32], with minor modifications. For iNOS (257 bp), TNF- $\alpha$ (275 bp), IL-6 (286 bp), CINC-1 (231 bp), IL-1RI (327 bp), and elongation factor-1 $\alpha$ (EF; internal control) (335 bp) mRNA, an oligo(dT) primer was used for RT and the indicated primers sets were used for PCR (Table 1).

For the antisense transcript of iNOS $(211$ bp $)$, the sense primer (5'-TGCCCCTCCCCCACATTCTCT-3') was used for RT and the indicated primer set was used for PCR (Table 1). These mRNAs and antisense transcript levels were measured with real-time RT-PCR using the Rotor-Gene Q 2plex HRM (Qiagen, Tokyo, Japan). The Rotor-Gene SYBR Green PCR Kit (Qiagen) was included in the reaction mixture and the following touchdown protocol was applied: one cycle at $95^{\circ} \mathrm{C}$ for $5 \mathrm{~min}$ and 45 cycles at $95^{\circ} \mathrm{C}$ for $5 \mathrm{~s}$ and $60^{\circ} \mathrm{C}$ for $10 \mathrm{~s}$. The cDNAs for the rat iNOS mRNA and antisense transcript were deposited in the DNA Data Bank of Japan/European Bioinformatics Institute (DDBJ/EMBL)/GenBank under the accession numbers AB250951 and AB250952 respectively. 
Electrophoretic mobility shift assay: Nuclear extracts were prepared and an electrophoretic mobility shift assay was performed as previously described [32]. Briefly, nuclear extracts from hepatocytes $(4 \mu \mathrm{g})$ were mixed with $1 \mu \mathrm{g}$ of poly $(\mathrm{dI}-\mathrm{dC})$ and a probe for $20 \mathrm{~min}$ at room temperature (total mixture, $15 \mu \mathrm{l}$ ). To prepare the double-stranded DNA probe, annealed oligonucleotides harboring a $\mathrm{\kappa B}$ site (5'-AGTTGAGGGGACTTTCCCAGGC-3'; only the sense strand is shown) were labelled with $\left[\gamma^{-32} \mathrm{P}\right]$-adenosine-5'-triphosphate (ATP; DuPont-New England Nuclear Japan, Tokyo, Japan) and T4 polynucleotide kinase (Takara Bio Inc., Shiga, Japan). Samples were resolved on a $4.8 \%$ polyacrylamide gel, followed by drying and autoradiography. Protein concentrations were measured using the Bradford method [33] with a binding assay kit (Bio-Rad) and bovine serum albumin was used as a standard.

Construction of luciferase reporter plasmids and expression plasmids: The 1.0-kilobase (kb) 5'-flanking region including a TATA box of rat iNOS gene was inserted to pGL3-Basic vector (Promega, WI, USA) to create pRiNOS-Luc-SVpA [32]. Rat cDNA for the 3'UTR of $\mathrm{mNOS}$ was amplified with the primers (5'-tgctctaGACAGTGAGGGGTTTGGAGAGA-3' 5'-gcggatcctttaTTCTTGATCAAACACTCATTTT-3'), and the resultant cDNA was digested with BamH I and Xba I. This cDNA for the iNOS 3'UTR (submitted to the DDBJ/EMBL/GenBank under accession No. AB250951) replaced the SV40 polyadenylation signal (SVpA) of pRiNOS-Luc to create pRiNOS-Luc-3'UTR [17].

Transfection and luciferase assay: Transfection of cultured hepatocytes was performed as previously described [34]. Briefly, hepatocytes were cultured at $3 \times 10^{5}$ cells/dish $(35 \times 10$ $\mathrm{mm}$ ) in WE supplemented with serum, dexamethasone, and insulin for $7 \mathrm{~h}$, before being subjected to magnet-assisted transfection. The reporter plasmid pRiNOS-Luc-SVpA or pRiNOS-Luc-3' UTR $(1 \mu \mathrm{g})$ and the CMV promoter-driven $\beta$-galactosidase plasmid pCMV-LacZ (1 ng) as an internal control were mixed with magnet-assisted transfection-A reagent ( $1 \mu \mathrm{l}$; IBA GmbH, Göttingen, Germany).

After incubation for $15 \mathrm{~min}$ on a magnetic plate at room temperature, the medium was replaced with fresh WE containing serum. Cells were cultured overnight and then treated with IL-1 $\beta$ in the presence or absence of GSH. Luciferase and $\beta$-galactosidase activities of cell extracts were measured using PicaGene (Wako Pure Chemicals) and Beta-Glo (Promega, Wisconsin, USA) kits, respectively.

Statistical analysis: The results shown are representative of three to four independent experiments that yielded similar findings. All data are expressed as the mean \pm SD. Differences were analyzed by the Bonferroni-Dunn test, and a value of $\mathrm{P}<0.05$ was considered to indicate a significant difference. 
Table 1. Primers and nucleotide sequences

\begin{tabular}{|c|c|}
\hline Primer name & Nucleotide sequence \\
\hline iNOS F/R & 5' CCAACCTGCAGGTCTTCGATG 3'/5' GTCGATGCACAACTGGGTGAAC 3' \\
\hline as iNOS F/R & 5' CCTTTGCCTCATACTTCCTCAGA 3'/5' ATCTTCATCAAGGAATTATACACGG 3' \\
\hline TNF- $\alpha \mathrm{F} / \mathrm{R}$ & 5' TCCCAACAAGGAGGAGAAGTTCC 3'/5' GGCAGCCTTGTCCCTTGAAGAGA 3' \\
\hline IL-6 F/R & 5' GAGAAAAGAGTTGTGCAATGGCA 3'/5' TGAGTCTTTTATCTCTTGTTTGAAG 3' \\
\hline CINC-1 F/R & 5' GCCAAGCCACAGGGGCGCCCGT 3’/5' ACTTGGGGACACCCTTTAGCATC 3' \\
\hline IL-1RI F/R & 5'-CGAAGACTATCAGTTTTTGGAAC-3'/5'-GTCTTTCCATCTGAAGCTTTTGG-3' \\
\hline $\mathrm{EF} F / \mathrm{R}$ & 5', TCTGGTTGGAATGGTGACAACATGC 3'/5' CCAGGAAGAGCTTCACTCAAAGCTT 3 ' \\
\hline
\end{tabular}

iNOS, inducible nitric oxide synthase; as, antisense transcript; TNF- $\alpha$, tumor necrosis factor-alpha; IL-6, interleukin-6; CINC-1, cytokine-induced neutrophil chemoattractant-1; IL-1R1, type I IL-1 receptor; EF, elongation factor- $1 \alpha$; $/ \mathrm{R}$, forward/reverse.

\section{RESULTS}

\section{GSH inhibits NO production and iNOS induction}

The proinflammatory cytokine IL-1 $\beta$ stimulated iNOS induction, which was followed by production of NO in primary cultured rat hepatocytes. Simultaneous addition of GSH and IL-1 $\beta$ reduced nitrite levels in a time and dose-dependent manner in culture medium (Fig. 1A and 1B). GSH showed more than $90 \%$ inhibition at $20-25 \mathrm{mg} / \mathrm{ml}$. GSH had no cellular cytotoxicity at various concentrations as observed by Trypan blue exclusion in hepatocytes (data not shown).

Western blotting analysis showed that GSH dose-dependently reduced iNOS protein expression, demonstrating its IC $_{50}$ at $15 \mathrm{mg} / \mathrm{ml}$ (Fig. 1B). Real-time RT-PCR analysis showed that GSH decreased iNOS mRNA expression (Fig. 1C). These results suggest that GSH inhibited induction of iNOS gene expression at the transcriptional and/or post-transcriptional level. Expression of other mRNAs was then examined. IL-1 $\beta$ increased the levels of proinflammatory cytokine expression, such as TNF- $\alpha$, CINC-1, and IL-6. GSH decreased TNF- $\alpha$, CINC-1, and IL-6 mRNA levels (Fig. 1D, 1E, and 1F). 

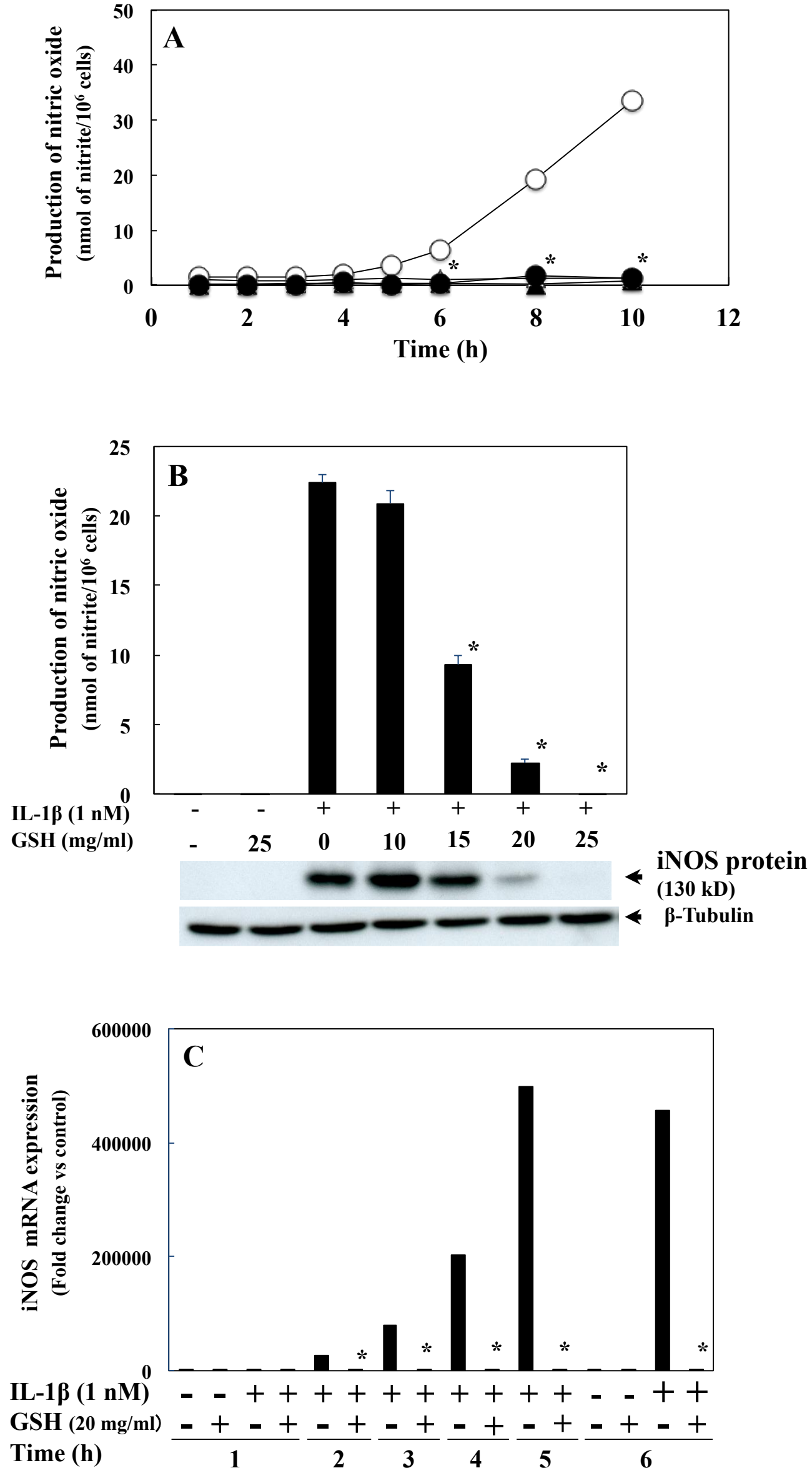

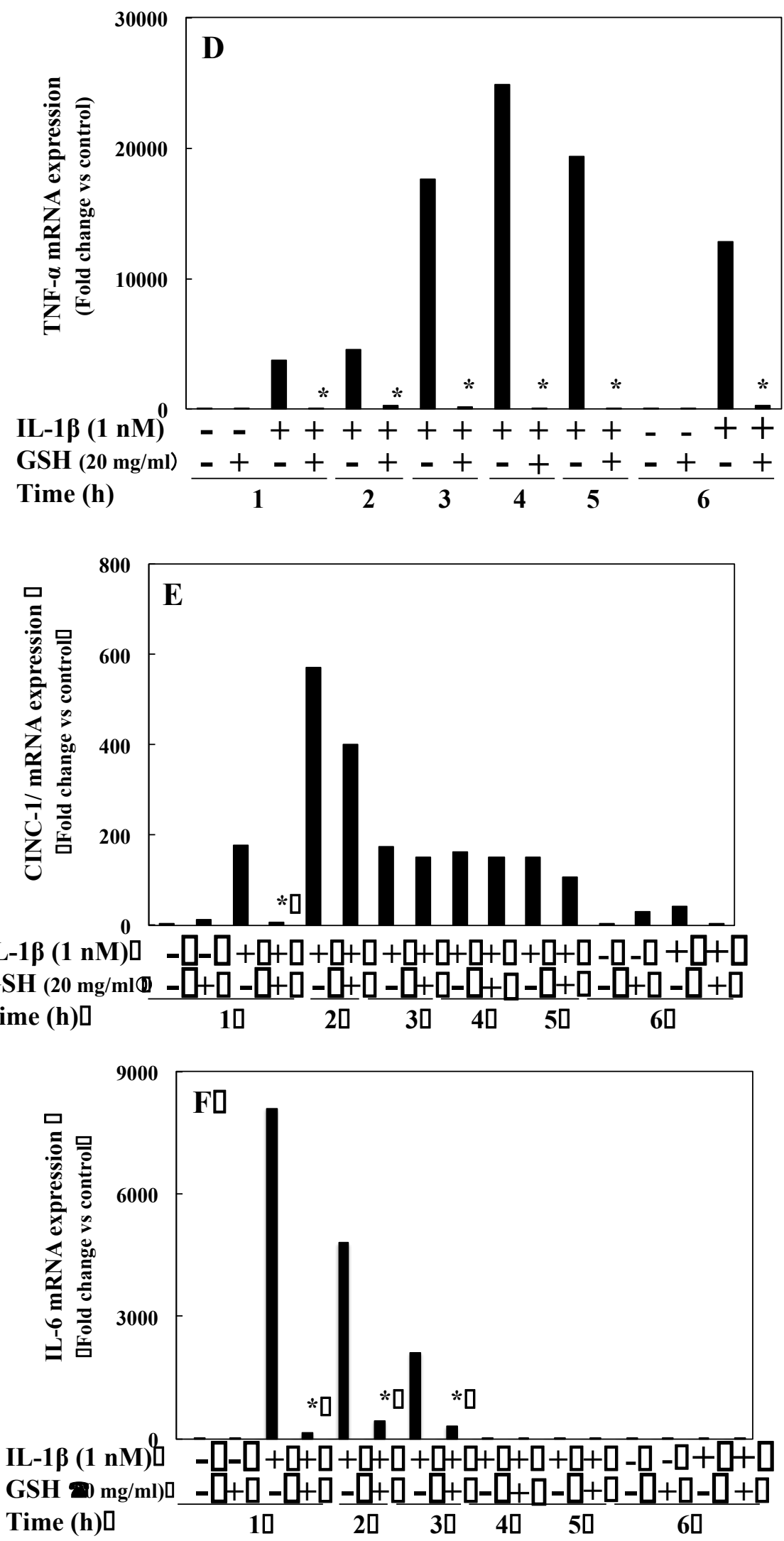

Figure 1. Effects of GSH on induction of NO production, and iNOS and mRNA expression of proinflammatory cytokines in IL-1 $\beta$-stimulated hepatocytes.

Cultured hepatocytes were treated with IL-1 $\beta(1 \mathrm{nM})$ in the presence or absence of GSH $(10-25 \mathrm{mg} / \mathrm{ml})$.

(A) Effect of GSH $(20 \mathrm{mg} / \mathrm{ml})$ treatment for the indicated times on NO production (IL-1 $\beta$, open circles; IL-1 $\beta$ + GSH, filled circles; GSH, filled triangles; controls [without IL-1 $\beta$ and GSH], open triangles). 
(B) Effects of treatment with various doses of GSH (10-25 mg/ml) for $8 \mathrm{~h}$ on NO production (upper panel) and iNOS protein levels (medium). Nitrite levels were measured in the culture medium. Data are presented as the mean \pm SD for $n=3$ dishes/point; $* \mathrm{P}<0.05$ versus IL-1 $\beta$ alone. In the western blot panels, cell lysates (20 $\mu$ g of protein) were subjected to SDS-PAGE in a $7.5 \%$ gel and immunoblotted with an anti-iNOS or anti- $\beta$-tubulin antibody.

(C) Effects of GSH $(20 \mathrm{mg} / \mathrm{ml})$ treatment for the indicated times on iNOS mRNA expression. Total RNA was analyzed by strand-specific RT-PCR to detect iNOS mRNA, using EF mRNA as an internal control. Total RNA was analyzed using strand-specific RT-PCR to detect

(D) TNF- $\alpha$, (E) CINC-1, and (F) IL-6 using EF mRNA as an internal control.

\section{GSH decreases iNOS mRNA synthesis and stabilization}

We examined the mechanisms that are involved in inhibiting iNOS induction. Expression of iNOS mRNA is regulated by iNOS promoter transactivation with transcription factors, such as NF- $\kappa \mathrm{B}$, and by post-transcriptional modifications, such as mRNA stabilization [35]. Therefore, we performed transfection experiments using constructs that contained firefly luciferase controlled by the iNOS promoters (pRiNOS-Luc-SVpA and pRiNOS-Luc-3' UTR) [17], which detect iNOS promoter transactivation (mRNA synthesis) and mRNA stabilization respectively. IL-1 $\beta$ increased luciferase activity of these two constructs, and these effects were significantly inhibited by GSH (Fig. 2A and 2B).
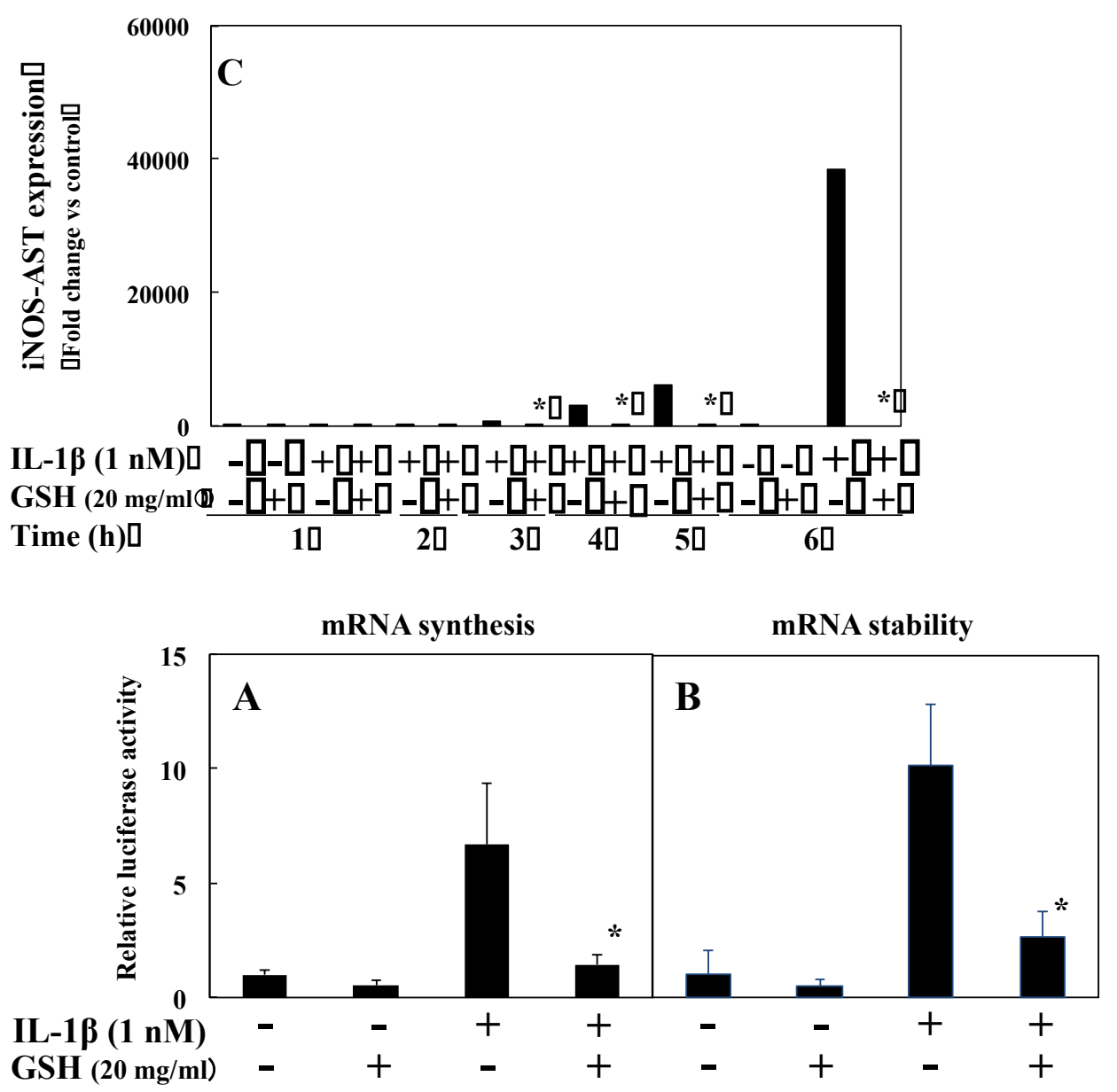

Figure 2. Effects of GSH on iNOS promoter transactivation and iNOS gene antisense transcript expression.

(A, B) Each construct was introduced into hepatocytes. The cells were treated with IL-1 $\beta$ (1 nM) in the 
presence or absence of GSH $(20 \mathrm{mg} / \mathrm{ml})$ for $7 \mathrm{~h}$ for pRiNOS-Luc-SVpA (A) and $3.5 \mathrm{~h}$ for pRiNOS-Luc- $3^{\prime}$

UTR (B). Luciferase activity was normalized by $\beta$-galactosidase activity. Fold activation was calculated by dividing luciferase activity by control activity (without IL-1 $\beta$ and GSH). Data are presented as the mean \pm SD for $\mathrm{n}=4$ dishes. $* \mathrm{P}<0.05$ versus IL-1 $\beta$ alone. (C) The cells were treated with IL-1 $\beta \quad(1 \mathrm{nM})$ in the presence or absence of GSH $(20 \mathrm{mg} / \mathrm{ml})$ for the indicated times. Total RNA was analyzed by strand-specific RT-PCR to detect the iNOS gene antisense transcript (asRNA).

Additionally, iNOS antisense transcript (asRNA) analysis using real-time RT-PCR showed that IL-1 $\beta$ increased iNOS asRNA expression in a time-dependent manner, and that GSH markedly inhibited this effect (Fig. 2C).

\section{GSH has no effect on NF- $\kappa$ B activation, but it inhibits IL-1RI upregulation}

There are two essential signaling pathways for induction of iNOS, called the IкB kinase and phosphatidylinositol 3-kinase (PI3K)/Akt pathways. In the IкB kinase pathway, IL-1 $\beta$ stimulates degradation of I $\mathrm{B}$ protein after phosphorylation by I $\kappa \mathrm{B}$ kinase, which is followed by activation of NF- $\kappa \mathrm{B}$ (i.e., translocation from the cytoplasm to the nucleus and DNA binding). An electrophoretic mobility shift assay with nuclear extracts showed that GSH did not inhibit NF- $\kappa \mathrm{B}$ activation at $1-4 \mathrm{~h}$, which indicated no blockade of NF- $\kappa \mathrm{B}$ nuclear translocation (Fig. 3B). GSH also had no effect on IкB degradation at the upper stream (Fig. $3 \mathrm{~A})$.

A

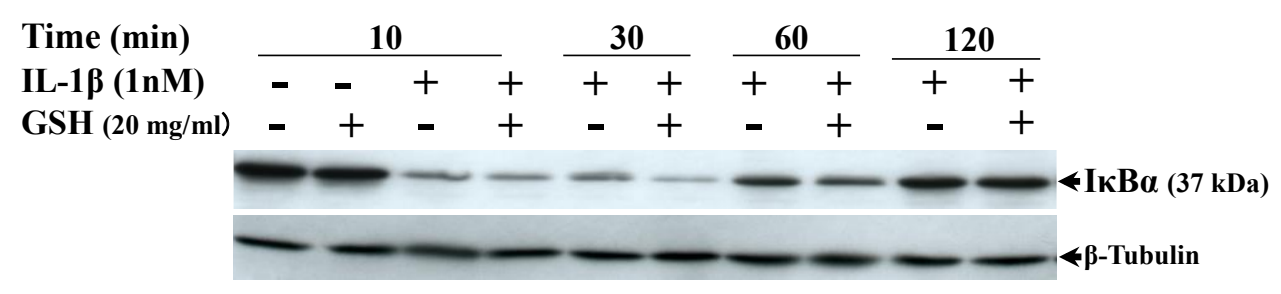

B

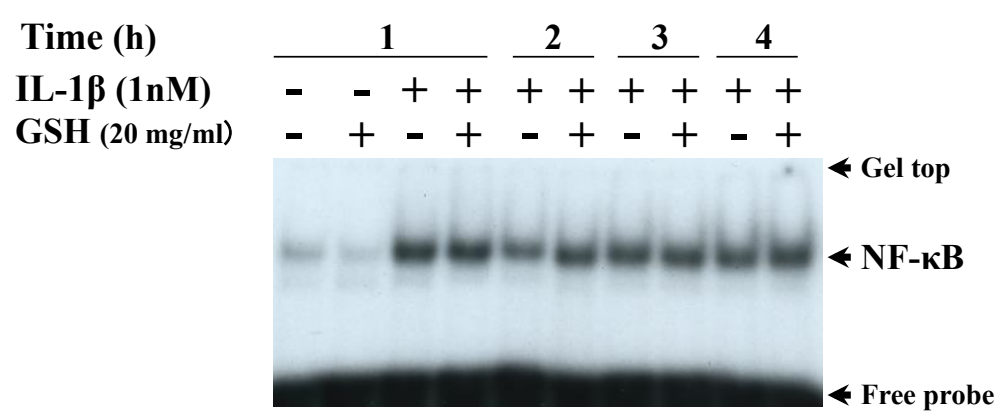

Figure 3. Effects of GSH on degradation of $\mathrm{I} \kappa \mathrm{B} \alpha$ and activation of NF- $\kappa \mathrm{B}$. Cells were treated with IL-1 $\beta \quad(1 \mathrm{nM})$ in the presence or absence of GSH $(20 \mathrm{mg} / \mathrm{ml})$ for the indicated times.

(A) Cell lysates $(20 \mu \mathrm{g}$ of protein) were subjected to SDS-PAGE in a $12.5 \%$ gel, followed by immunoblotting with an anti-I $\kappa \mathrm{B} \alpha$ or anti- $\beta$-tubulin antibody. (B) Activation of NF- $\kappa$ B. Nuclear extracts (4 $\mu \mathrm{g}$ of protein) were analyzed using an electrophoretic mobility shift assay. Representative results of three independent experiments are shown. 
In the PI3K/Akt pathway, IL-1 $\beta$ stimulates upregulation of IL-1RI by activation of PI3K/Akt [36]. Immunoprecipitation-western blotting analysis showed that GSH inhibited phosphorylation (activation) of Akt, a downstream kinase of PI3K (Fig. 4A). Real-time RT-PCR and western blotting analyses showed that GSH reduced IL-1RI mRNA expression levels (Fig. 4B).

A
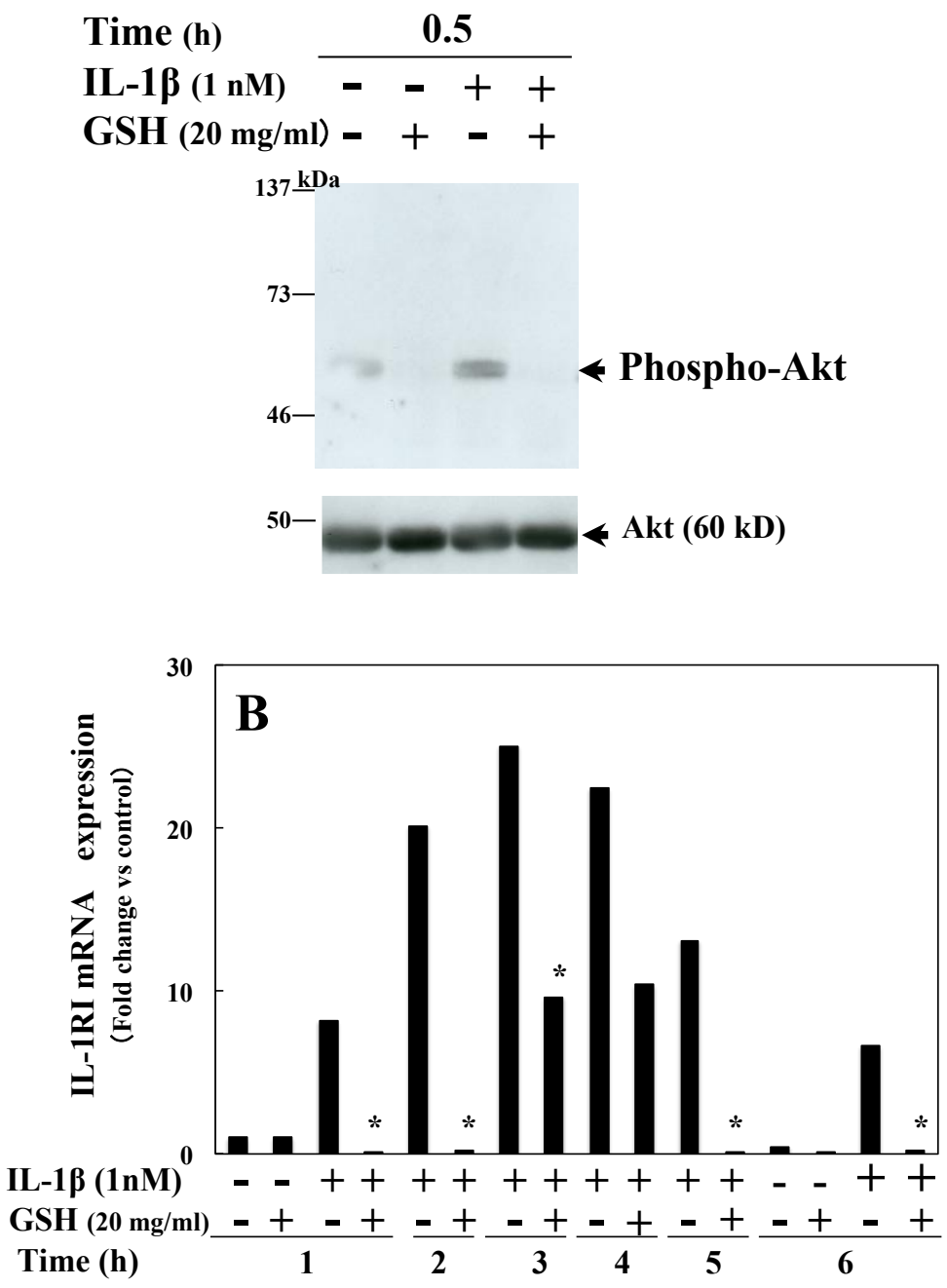

Figure 4. Effects of GSH on upregulation of IL-1RI. Cells were treated with IL-1 $\beta$ (1 nM) in the presence or absence of GSH $(20 \mathrm{mg} / \mathrm{ml}))$ for the indicated times.

(A) Phosphorylation of Akt. Total cell lysates were immunoprecipitated with an anti-Akt antibody, followed by immunoblotting (SDS-PAGE in a gel with a gradient of 6\%-9\%) with an anti-phospho-Akt or anti-Akt antibody. (B) Total RNA was analyzed using strand-specific RT-PCR to detect IL-1RI mRNA, using EF mRNA as an internal control.

\section{Delayed administration of GSH inhibits iNOS induction}

We examined whether delayed administration of GSH affects induction of iNOS. GSH (20 $\mathrm{mg} / \mathrm{ml}$ )) was added to the medium $0-5 \mathrm{~h}$ after addition of IL-1 $\beta$. Delayed administration of GSH up to $5 \mathrm{~h}$ after IL-1 $\beta$ addition still markedly inhibited NO production, although the magnitude of inhibition decreased in a time-dependent manner (Fig. 5). 


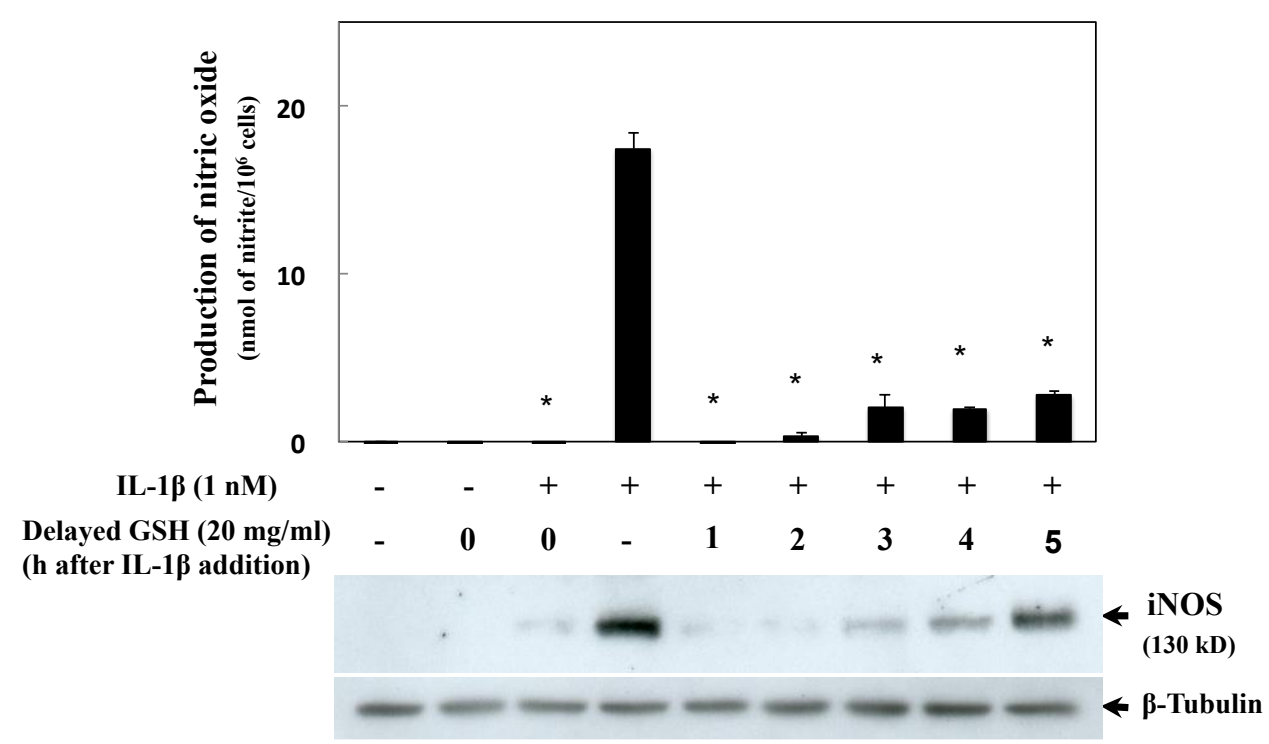

Figure 5. Effects of delayed GSH administration on induction of iNOS in hepatocytes. Cells were treated with GSH $(20 \mathrm{mg} / \mathrm{ml}))$ at $0-5 \mathrm{~h}$ after addition of IL-1 $\beta(1 \mathrm{nM})$.

The effects of GSH on NO production (upper panel) and iNOS protein (middle) were analyzed at $8 \mathrm{~h}$ after the addition of IL-1 $\beta$. Nitrite levels were measured in the culture medium. Data are presented as the mean $\pm \mathrm{SD}, \mathrm{n}$ $=3$ dishes/point; $* \mathrm{P}<0.05$ versus IL-1 $\beta$ alone. In the western blotting panels, cell lysates (20 $\mu \mathrm{g}$ of protein) were subjected to SDS-PAGE in a 7.5\% gel and immunoblotted with an anti-iNOS or anti- $\beta$-tubulin antibody.

\section{DISCUSSION}

GSH is a bioactive peptide and important non-enzymatic antioxidant that is widely present in living organisms [10]. The highest levels of GSH appear in the liver, which is the major organ for GSH synthesis and metabolism. GSH can promote metabolism of sugar, fat, and protein, and maintains normal cell metabolism and cell membrane integrity. GSH can bind toxic substances, such as electrophilic radicals and oxygen free radicals, and has extensive antioxidative effects [10]. Currently, GSH preparations are widely used for treating certain liver diseases, including viral hepatitis, liver cirrhosis, and drug-induced liver injury [10, 37, 38].

In the current study, we used primary cultured rat hepatocytes that were stimulated by the proinflammatory cytokine IL-1 $\beta$ (in vitro liver injury model) [27] to investigate the effects and mechanisms of GSH in the injured liver. We found that GSH inhibited induction of iNOS and its mRNA and protein expression, thereby preventing NO production (Fig. 1A, $1 \mathrm{~B}$, and $1 \mathrm{C})$. Additionally, in transfection experiments with iNOS promoter constructs (containing two NF- $\kappa$ B binding sites), GSH decreased iNOS mRNA levels by inhibiting its mRNA synthesis and stabilization steps (Fig. 2A and 2B). For iNOS mRNA stabilization, GSH decreased iNOS asRNA expression (Fig. 2C). We recently reported that asRNAs are often transcribed from many inducible genes, such as iNOS and TNF- $\alpha$ [34]. Furthermore, iNOS asRNA interacts with and stabilizes iNOS mRNA [39, 40].

Proinflammatory cytokines, such as TNF- $\alpha$, IL-1 $\beta$, and IL-6, play an important role in injury to multiple organs in addition to the liver. In our study, GSH also inhibited TNF- $\alpha$, 
CINC-1, and IL-6 mRNA expression (Fig. 1D, 1E, and 1F). These results suggested that GSH markedly inhibited the inflammatory pathway.

GSH had no effect on NF- $\kappa B$ activation in the electrophoretic mobility shift assay (Fig. 3B). In contrast, we found that GSH inhibited IL-1RI upregulation (Fig. 4B) by reducing Akt phosphorylation (Fig. 4A). Transfection experiments with iNOS promoter luc-constructs containing two NF- $\kappa \mathrm{B}$ binding sites (Fig. 2A and 2B) showed that GSH inhibited the activities of both constructs. Therefore, we cannot rule out the possibility that GSH can inhibit promoter transactivation via NF- $\kappa B$, although GSH had no effect in electrophoretic mobility shift assays. Various inflammatory genes, including iNOS, TNF- $\alpha$, and CINC-1, are regulated by transcription factor NF- $\mathrm{BB}$. We speculate that inactivation of $\mathrm{NF}-\kappa \mathrm{B}$ might be involved in mechanisms of decreased mRNA expression of these genes.

Delayed GSH treatment after IL-1 $\beta$ addition also caused a marked reduction in iNOS induction and NO production (Fig. 5). This observation may be of clinical importance because initiation of therapeutic GSH treatment is usually delayed from the onset of disease. Appropriate regulation of inflammatory reactions is essential in preventing the onset of organ damage and early recovery.

Our simple in vitro model with cultured hepatocytes may be adequate for screening of liver-protective drugs, because it is rapid and inexpensive compared with in vivo animal models of liver injury. However, the doses $(20 \mathrm{mg} / \mathrm{ml} ; 65 \mathrm{mM})$ used in the experiments with cultured hepatocytes are relatively high compared with intracellular levels, which is a limitation in this model. Furthermore, the liver-protective effects of drugs deduced from this model need to be examined and supported in in vivo animal models and other models. GSH has been administered by orally and via injection. In clinical practice in Japan, GSH is used in both oral preparations and injectable preparations, which are reduced formulations. It is mainly used for drug and metal poisoning, such as from Arsenic. Because it is used for poisoning symptoms, it is often injected as an emergency. Additionally, the organism has a defense mechanism by antioxidant against reactive oxygen species and the reduced GSH is consumed [41, 42]. Yamada et al. described that most of orally administered glutathione in portal blood is oxidized glutathione [43]. Therefore, we examined GSH with the assumption reduced GSH injection pathway in "in vitro liver injury model".

In conclusion, GSH can prevent IL-1 $\beta$-stimulated liver injury in cultured hepatocytes by inhibiting induction of inflammatory mediators, such as iNOS and TNF- $\alpha$. GSH may have therapeutic potential for treating liver injury.

List of Abbreviations: GSH, glutathione; iNOS, inducible nitric oxide synthase; TNF- $\alpha$, tumor necrosis factor-alpha; NO, nitric oxide; IL, interleukin; NF- $\mathrm{BB}$, nuclear factor-kappa B; CINC-1, cytokine-induced neutrophil chemoattractant-1; SDS-PAGE, sodium dodecyl sulfate-polyacrylamide gel electrophoresis; IL-1RI, type I interleukin-1 receptor; RT-PCR, reverse transcriptase-polymerase chain reaction

Competing Interests: The authors declare that there are no conflicts of interest. 
Authors' Contributions: RN participated in the design of the study, data collection, statistical analysis, and drafting of the manuscript. TO participated in supervision and provided oversight when the manuscript was being drafted. MK, HY, MH, MI, KM, and MK assisted in the design of the study. MN provided advice regarding development of the protocol for the study and assisted in the design of the study.

Acknowledgments and Funding: This work was supported in part by a Grant-in-Aid for Scientific Research from the Ministry of Education, Science, Culture and Sports of Japan, and by grants from the Science Research Promotion Fund of the Japan Private School Promotion Foundation. Cooper AJL: Glutathione in the brain: disorders of glutathione metabolism. In The Molecular and Genetic Basis of Neurological Disease. Edited by Rosenberg RN, Prusiner SB, DiMauro S, Barchi RL and Kunk LM. Boston: Butterworth-Heinemann; 1997:1195-1230.

\section{REFERENCES}

1. Dringen R: Metabolism and functions of glutathione in brain. Prog Neurobiol 2000, 62: 649-671.

2. Droge W, Schulze-Osthoff K, Mihm S, Galter D, Schenk H, et al.: Functions of glutathione and glutathione disulfide in immunology and immunopathology. FASEB J 1994, 8: 1131-1138.

3. Fischman CM, Udey MC, Kurtz M, Wedner HJ: Inhibition of lectin-induced lymphocyte activation by 2-cyclohexene-1-one: decreased intracellular glutathione inhibits an early event in the activation sequence. J Immunol 1981, 127: 2257-2262.

4. Hamilos DL, Zelarney P, Mascali JJ: Lymphocyte proliferation in glutathione-depleted lymphocytes: direct relationship between glutathione availability and the proliferative response. Immunopharmacology 1989, 18 : 223-235.

5. Ishii T, Sugita Y, Bannai S: Regulation of glutathione levels in mouse spleen lymphocytes by transport of cysteine. J Cell Physiol 1987, 133: 330-336.

6. Satz GT, Bannister WH, Bannister JV: Free radicals and thiol compounds: the role of glutathione against free radical toxicity. In Glutathione: Metabolism and Physiological Functions. Edited by Vina J. Boca Raton: CRC Press; 1990: 237-257.

7. Kim JM, Kim H, Kwon SB, Lee SY, Chung SC, et al.: Intracellular glutathione status regulates mouse bone marrow monocyte-derived macrophage differentiation and phagocytic activity. Biochem Biophys Res Commun 2004, 325:101-108.

8. Sido B, Hack V, Hochlehnert A, Lipps H, Herfarth C, et al.: Impairment of intestinal glutathione synthesis in patients with inflammatory bowel disease. Gut 1998, 42:485-492. 
9. Chen Y, Dong H, Thompson DC, Shertzer HG, Nebert DW, et al.: Glutathione defense mechanism in liver injury: insights from animal models. Food Chem Toxicol 2013, 60: 38-44.

10. Colasanti M, Suzuki H: The dual personality of NO. Trends in Pharmacological Sciences 2000, 21: 249-252.

11. Mantena SK, Vaughn DP, Andringa KK, Eccleston HB, King AL, et al.: High fat diet induces dysregulation of hepatic oxygen gradients and mitochondrial function in vivo. Biochem J 2009, 417: 183-193.

12. Raso GM, Esposito E, Iacono A, Pacilio M, Cuzzocrea S, et al.: Comparative therapeutic effects of metformin and vitamin $\mathrm{E}$ in a model of non-alcoholic steatohepatitis in the young rat. Eur J Pharmacol 2009, 604: 125-131.

13. Anavi S, Eisenberg-Bord M, Hahn-Obercyger M, Genin O, Pines M, et al.: The role of iNOS in cholesterol-induced liver fibrosis. Lab Invest 2015, 95: 914-924.

14. Tsuchiya H, Kaibori M, Yanagida H, Yokoigawa N, Kwon AH, et al.: Pirfenidone prevents endotoxin-induced liver injury after partial hepatectomy in rats. J Hepatol 2004, 40: 94-101.

15. Tsuji K, Kwon AH, Yoshida H, Qiu Z, Kaibori M, et al.: Free radical scavenger (edaravone) prevents endotoxin-induced liver injury after partial hepatectomy in rats. J Hepatol 2005, 42: 94-101.

16. Tanaka H, Uchida Y, Kaibori M, Hijikawa T, Ishizaki M, et al.: Na+/H+ exchanger inhibitor, FR183998, has protective effect in lethal acute liver failure and prevents iNOS induction in rats. J Hepatol 2008, 48: 289-299.

17. Ishizaki M, Kaibori M, Uchida Y, Hijikawa T, Tanaka H, et al.: Protective effect of FR183998, a Na+/H+ exchanger inhibitor, and its inhibition of iNOS induction in hepatic ischemia-reperfusion injury in rats. Shock 2008, 30: 311-317.

18. Hijikawa T, Kaibori M, Uchida Y, Yamada M, Matsui K, et al.: Insulin-like growth factor 1 prevents liver injury through the inhibition of TNF-alpha and iNOS induction in D-galactosamine and LPS-treated rats. Shock 2008, 29: 740-747.

19. Nakatake R, Tanaka Y, Ueyama Y, Miki H, Ishizaki M, et al.: Protective effects of active hexose correlated compound in a rat model of liver injury after hepatectomy. Functional Foods in Health and Disease 2016, 6: 702-717.

20. Nakanishi H, Kaibori M, Teshima S, Yoshida H, Kwon AH, et al.: Pirfenidone inhibits the induction of iNOS stimulated by interleukin-1beta at a step of NF-kappaB DNA binding in hepatocytes. J Hepatol 2004, 41: 730-736.

21. Yoshida H, Kwon AH, Kaibori M, Tsuji K, Habara K, et al.: Edaravone prevents NOS expression by inhibiting its promoter transactivation and mRNA stability in cytokine-stimulated hepatocytes. Nitric Oxide 2008, 18: 105-112. 
22. Nakatake R, Iida H, Ishizaki M, Matsui K, Nakamura Y, et al.: Metformin inhibits expression of the proinflammatory biomarker inducible nitric oxide synthase in hepatocytes. Functional Foods in Health and Disease 2018, 8: 175-192.

23. Nakatake R, Kaibri M, Miki H, Tsuda T, Ueyama Y, et al: Glutamic acid has a liver-protective effect through the suppression of inducible nitric oxide synthase in primary cultured rat hepatocytes. IJARCS 2015, 2: 47-63.

24. Nakatake R, Hishikawa H, Matushima H, Nakamura Y, Ishizaki M, Matsui K, Kaibori M, et al.: Curcumin protects liver inflammation by suppressing expression of inducible nitric oxide synthase in primary cultured rat hepatocytes. Functional Foods in Health and Disease 2017, 7: 716-734.

25. Matsui K, Kawaguchi Y, Ozaki T, Tokuhara K, Tanaka H, et al.: Effect of active hexose correlated compound on the production of nitric oxide in hepatocytes. JPEN J Parenter Enteral Nutr 2007, 31: 373-380; discussion 380-371.

26. Kaibori M, Okumura T, Sato K, Nishizawa M, Kon M: Inducible nitric oxide synthase expression in liver injury: liver protective effects on primary rat hepatocytes. Inflamm Allergy Drug Targets 2015, 14: 77-83.

27. Seglen PO: Preparation of isolated rat liver cells. Methods Cell Biol 1976, 13: 29-83.

28. Horiuti Y, Ogishima M, Yano K, Shibuya Y: Quantification of cell nuclei isolated from hepatocytes by cell lysis with nonionic detergent in citric acid. Cell Struct Funct 1991, 16: 203-207.

29. Green LC, Wagner DA, Glogowski J, Skipper PL, Wishnok JS, et al.: Analysis of nitrate, nitrite, and [15N]nitrate in biological fluids. Anal Biochem 1982, 126 : 131-138.

30. Chomczynski P, Sacchi N: Single-step method of RNA isolation by acid guanidinium thiocyanate-phenol-chloroform extraction. Anal Biochem 1987, 162:1 56-159.

31. Oda M, Sakitani K, Kaibori M, Inoue T, Kamiyama Y, et al.: Vicinal dithiol-binding agent, phenylarsine oxide, inhibits inducible nitric-oxide synthase gene expression at a step of nuclear factor-kappaB DNA binding in hepatocytes. J Biol Chem 2000, 275: 4369-4373.

32. Bradford MM: A rapid and sensitive method for the quantitation of microgram quantities of protein utilizing the principle of protein-dye binding. Anal Biochem 1976, 72: 248-254.

33. Nishizawa M, Ikeya Y, Okumura T, Kimura T: Post-transcriptional inducible gene regulation by natural antisense RNA. Front Biosci 2015, 20: 1-39. 
34. Kleinert H, Pautz A, Linker K, Schwarz PM: Regulation of the expression of inducible nitric oxide synthase. Eur J Pharmacol 2004, 500:255-266.

35. Teshima S, Nakanishi H, Nishizawa M, Kitagawa $K$, Kaibori $M$, et al.: Up-regulation of IL-1 receptor through PI3K/Akt is essential for the induction of iNOS gene expression in hepatocytes. J Hepatol 2004, 40: 616-623.

36. Balendiran GK, Dabur R, Fraser D: The role of glutathione in cancer. Cell Biochem Funct 2004, 22: 343-352.

37. Lin Y, Li Y, Hu X, Liu Z, Chen J, et al.: The hepatoprotective role of reduced glutathione and its underlying mechanism in oxaliplatin-induced acute liver injury. Oncol Lett 2018, 15: 2266-2272.

38. Matsui K, Nishizawa M, Ozaki T, Kimura T, Hashimoto I, et al.: Natural antisense transcript stabilizes inducible nitric oxide synthase messenger RNA in rat hepatocytes. Hepatology 2008, 47: 686-697.

39. Yoshigai E, Hara T, Araki Y, Tanaka Y, Oishi M, et al.: Natural antisense transcript-targeted regulation of inducible nitric oxide synthase mRNA levels. Nitric Oxide 2013, 30: 9-16.

40. Pigeolet E, Corbisier P, Houbion A, Lambert D, Michiels C et al.: Glutathione peroxidase, superoxide dismutase, and catalase inactivation by peroxides and oxygen derived free radicals. Mech Ageing Dev 1990, 51: 283-297.

41. Sakurai T, Ochiai M, Kojima C, Ohta T, Sakurai MH et al.: Preventive mechanism of cellular glutathione in monomethylarsonic acid-induced cytolethality. Toxicol Appl Pharmacol 2005, 206(1): 54-65.

42. Hiroaki Yamada, Shinn Ono, Sayori Wada, Wataru Aoi, Eun Young Park et al.: Statuses of food-derived glutathione in intestine, blood, and liver of rat. Science of Food 2018, 2: Article number 3. 